Article

\title{
Two MYB and Three bHLH Family Genes Participate in Anthocyanin Accumulation in the Flesh of Peach Fruit Treated with Glucose, Sucrose, Sorbitol, and Fructose In Vitro
}

\author{
Jiao Wang ${ }^{1,2}$, Ke Cao ${ }^{2}$, Lirong Wang ${ }^{2}$, Wenxuan Dong ${ }^{1}$, Xiao Zhang ${ }^{1}{ }^{\mathbb{D}}$ and Weisheng Liu ${ }^{1, *}$ \\ 1 College of Horticulture, Shenyang Agricultural University, Shenyang 110866, China; \\ wangiiaolingling@163.com (J.W.); dongwx63@syau.edu.cn (W.D.); zhangxiao8866@syau.edu.cn (X.Z.) \\ 2 The Key Laboratory of Biology and Genetic Improvement of Horticultural Crops (Fruit TreeBreeding \\ Technology), Ministry of Agriculture, Zhengzhou Fruit Research Institute, Chinese Academy of Agricultural \\ Sciences, Zhengzhou 450009, China; caoke@caas.cn (K.C.); wanglirong@caas.cn (L.W.) \\ * Correspondence: wsliulaas@163.com
}

check for updates

Citation: Wang, J.; Cao, K.; Wang, L.; Dong, W.; Zhang, X.; Liu, W. Two MYB and Three bHLH Family Genes Participate in Anthocyanin Accumulation in the Flesh of Peach Fruit Treated with Glucose, Sucrose, Sorbitol, and Fructose In Vitro. Plants 2022, 11, 507. https://doi.org/ $10.3390 /$ plants 11040507

Academic Editor: Dariusz Latowski

Received: 29 November 2021

Accepted: 10 February 2022

Published: 13 February 2022

Publisher's Note: MDPI stays neutral with regard to jurisdictional claims in published maps and institutional affiliations.

Copyright: (C) 2022 by the authors. Licensee MDPI, Basel, Switzerland. This article is an open access article distributed under the terms and conditions of the Creative Commons Attribution (CC BY) license (https:// creativecommons.org/licenses/by/ $4.0 /)$.

\begin{abstract}
Anthocyanins are important pigments in peach fruit and are beneficial to human health. Sugars are both energy-storing and signaling molecules and their roles in inducing anthocyanin biosynthesis have received a great deal of research attention. However, the mechanism by which sugars induce anthocyanin biosynthesis in peach fruit is unknown. In order to understand this induction mechanism, comprehensive transcriptome and metabolome were performed in fruit flesh treated with four different sugars for 12 and $24 \mathrm{~h}$, respectively. Here, we found that cyanidin-3$\mathrm{O}-(6-\mathrm{O}$-p-coumaroyl) glucosides accumulated in fruit flesh treated with glucose, sucrose, sorbitol, and fructose in vitro. Two key structural genes of the anthocyanin biosynthesis pathway, namely, PpDFR and P $U$ UFGT, were upregulated in the flesh of sugar-treated peach fruit. By contrast, the two main transcription factors (TFs) $P p M Y B 10.1$ and $P p B L$ regulating anthocyanin biosynthetic genes in peach fruit were not upregulated accordingly. Interestingly, two MYB family genes (PpMYB6 and PpMYB44-like) and three bHLH family genes (PpbHLH35, PpbHLH51, and PpbHLH36-like) were upregulated. A dual luciferase assay revealed that PpMYB6 strongly activated the PpUFGT promoter when it was co-infiltrated with PpbHLH35, PpbHLH51, and PpbHLH36-like. When PpMYB44-like was co-infiltrated with PpbHLH35, it also potently activated the PpUFGT promoter. The results of this study help clarify the molecular mechanisms by which glucose, sucrose, sorbitol, and fructose regulate anthocyanin accumulation in peach fruit.
\end{abstract}

Keywords: anthocyanin; bHLH TF; dual luciferase assay; MYB TF; sugar treatment

\section{Introduction}

Anthocyanins are flavonoid end-products and are abundant in leaves, flowers, fruits, and seeds. Their colors range from red to blue-purple in these plant organs [1,2]. The anthocyanin core structures are aglycones and include pelargonidin, cyanidin, delphinidin, peonidin, and malvidin [3,4]. Anthocyanins cause red pigmentation which improves fruit appearance and attracts insects and animals that propagate seeds $[5,6]$. They also play vital roles in plant resistance to biotic stress (such as pest insect infestations and microbial pathogen infection) and abiotic stress (such as drought and salinity) [7].

Anthocyanins are synthesized in the cytoplasm and then vacuolated via glutathione S-transferase (GST) [8]. Anthocyanin biosynthesis is catalyzed by a multi-enzyme complex including phenylalanine ammonia-lyase (PAL), cinnamate-4-hydroxylase $(\mathrm{C} 4 \mathrm{H})$, 4coumaroyl:CoA-ligase (4CL), chalcone synthase (CHS), chalcone flavanone isomerase $(\mathrm{CHI})$, flavanone 3-hydroxylase $(\mathrm{F} 3 \mathrm{H})$, flavonoid $3^{\prime}$-hydroxylase $\left(\mathrm{F} 3^{\prime} \mathrm{H}\right)$, dihydroflavonol 4-reductase (DFR), leucoanthocyanidin dioxygenase (LDOX), UDP-glucose: flavonoid 3-O-glucosyltransferase (UFGT), and glutathione S-transferase (GST) [9]. 
Anthocyanin biosynthesis is regulated at the transcriptional level by R2R3-myeloblastosis (MYB), basic helix-loop-helix (bHLH), and WD40 that form the MBW complex [8]. In apples, the MYB transcription factors MdMYB10 and MdMYB110a control anthocyanin accumulation by forming homodimers with MdbHLH [10]. In grape, several MYB transcription factors have been identified, such as VvMYBA1-1, VvMYBA1-2, VvMYBA1, and VvMYBA2. Of these, VvMYBA1 and VvMYBA2 regulate anthocyanin accumulation in the fruit skin [11,12]. In citrus, the MYB transcription factor CcRuby plays an important role in the red flesh trait [13]. PyMYB10, PyMYB114, and PybHLH3 in pear [14,15], FvMYB10 and FvbHLH33 in wild strawberry [16], and AtMYB75 (PAP1), AtMYB90 (PAP2), AtMYB113, and AtMYB114 in Arabidopsis [9,17] participate in anthocyanin accumulation.

Sugars are both energy-storing and signaling molecules and their roles in promoting anthocyanin biosynthesis have received extensive research attention. The shoots of Clematis pitcheri cultured in the presence of high sucrose concentrations and nitrogen accumulated high anthocyanin levels [18]. Exogenous sucrose induced anthocyanin accumulation more effectively than a 1:1 fructose: glucose mixture in the hypocotyls and roots of red radish seedlings [19]. Glucose more effectively induced anthocyanin accumulation than sucrose and without exogenous sugar treatment in blackberry fruit [20]. Strawberry fruit treated with sucrose displayed high levels of pelargonidin derivatives and upregulation of the genes participating in the phenylpropanoid and flavonoid pathways [21]. In Arabidopsis, sucrose induces anthocyanin accumulation in a concentration-dependent manner [22].

Anthocyanins accumulate in two ways during peach fruit development. First, peak anthocyanin content occurred at the late stages of fruit development in the blood-flesh peach cultivars 'Beijingyixianhong', 'Wuyuexian', and 'Tianjin Shui Mi'. The associated anthocyanin biosynthesis-encoding genes PpPAL, P $\mathrm{UFGT}$, PpCHI, PpF3H, PpDFR, PpANS, and $P p C H S$ reached their highest transcription levels during the early-to-middle stages of fruit development [2,23]. Second, the anthocyanin content reached its highest levels during the early stages of fruit development in the blood-flesh peach cultivars 'Heiyoutao,' 'Sanguine Pilat,' and 'Sanguine Vineuse.' The aforementioned anthocyanin biosynthesis-encoding genes had lower transcription levels in these cultivars than they did in 'Beijingyixianhong' and 'Wuyuexian' $[23,24]$. Both anthocyanin accumulation mechanisms are determined by two alleles. The former is determined by the NAC family gene $P p B L$ mapped atop linkage group five. PpBL and MYB10.1 promote the transcription of the anthocyanin biosynthesisregulating genes which leads to anthocyanin accumulation in blood-flesh fruit $[25,26]$. The latter is determined by a recessive locus $(b f)$ mapped to linkage group four [23]. However, cyanidin-3-glucoside was the main anthocyanin component in both types of blood-flesh peach fruit.

Glucose, sucrose, sorbitol and fructose are the major sugar compositions in peach fruit [27]. Sugar induction of anthocyanin in fruit is very important, it will provide a scientific basis to promote the accumulation of anthocyanin in fruits, and also an important way to achieve the goal of regulating fruit color development. Studies on induction of peach fruit by different sugars have been reported. For example, sucrose more effectively stimulated anthocyanin accumulation than either glucose or fructose in media-cultured red leaf peach shoots [28]. In the 'Tenshin Suimitsuto' blood-flesh peach fruit cultivar, $100 \mathrm{mM}$ sucrose induced anthocyanin biosynthesis [29].

In summary, the anthocyanins biosynthesis and regulation have been studied very thoroughly in peach and other species. Moreover, sugar as signaling molecules also have been received extensive attention in promoting anthocyanin biosynthesis in many horticultural crops. However, few studies have been focused on peach anthocyanins induction by exogenous sugars and the corresponding induction mechanism. In the present study, we mainly focused on how different sugars induce anthocyanins accumulation in peach fruit flesh. We subjected the blood-flesh peach fruit cultivar 'Tianjin ShuiMi' to various sugar treatments. We found two MYB family genes: PpMYB6 (Prupe.5G065500), PpMYB44-like (Prupe.8G134900), and three bHLH family genes: Pp bHLH35 (Prupe.1G074400), Pp bHLH51 (Prupe.2G252600) and Pp bHLH36-like (Prupe.3G131500). (https://www.rosaceae.org/, (ac- 
cessed on 12 July 2021)). Functional analysis indicates that these TFs have the potential in promoting anthocyanin biosynthesis in four different sugar treated fruits. This will help clarify the molecular mechanisms by which glucose, sucrose, sorbitol, and fructose regulate anthocyanin accumulation in peach fruit. PpMYB6PpMYB44-likePpbHLH35PpbHLH51PpbHLH36-like

\section{Results}

\subsection{Levels of Metabolites Involved in Anthocyanin Biosynthesis}

There were 24,228 metabolites in the flesh of peach fruit treated with sugars. Of these, 3923, 3091, 4038, and 3938 were upregulated, while 7537, 7699, 8067, and 7807 were downregulated in the flesh of peach fruit treated with glucose, sucrose, sorbitol, and fructose, respectively, for $12 \mathrm{~h}$. Moreover, 5023, 5062, 4952, and 5490 were upregulated while 7602, 7444, 7734, and 7870 were downregulated in the flesh of peach fruit treated with glucose, sucrose, sorbitol, and fructose, respectively, for $24 \mathrm{~h}$ (Tables S1 and S2). The top 100 upregulated metabolites in the flesh of peach fruit treated with sugar for $12 \mathrm{~h}$ were associated with 'Biosynthesis of secondary metabolites', 'Metabolic pathways', and 'Flavonoid biosynthesis', while the top 100 downregulated metabolites in the flesh of peach fruit treated with sugar for $12 \mathrm{~h}$ were associated with 'Biosynthesis of antibiotics', 'Metabolic pathways', and 'Zeatin biosynthesis' (Figures S1, S2 and Tables S9-S16). Of all these metabolites detected from LCMS and high-resolution tandem mass spectrometer, the top 100 upregulated metabolites in the flesh of peach fruit treated with sugar for $24 \mathrm{~h}$ were associated with 'Biosynthesis of secondary metabolites', 'Metabolic pathways', 'Biosynthesis of phenylpropanoids', 'Flavone and flavonol biosynthesis', 'Isoflavonoid biosynthesis', and 'Flavonoid biosynthesis', while the top 100 downregulated metabolites in the flesh of peach fruit treated with sugar for $24 \mathrm{~h}$ were associated with 'Biosynthesis of antibiotics', 'Biosynthesis of secondary metabolites', and 'Metabolic pathways' (Figures S3, S4 and Tables S17-S24).

As the sugar-treated fruit was picked from the 'Tianjin Shui Mi' blood-fleshed peach landrace, we focused mainly on flavonoids in the anthocyanin biosynthetic pathway. Figure 1, Tables S3 and S4 shows that the metabolites dihydroquercetin and cyanidin-3-O(6-O-p-coumaroyl) glucoside were upregulated markedly in flesh samples of peach fruit treated with glucose, sucrose, sorbitol, and fructose for $12 \mathrm{~h}$ and $24 \mathrm{~h}$. The p-coumaroyl CoA, chalcone, and cyanidin 3-O-glucoside levels were lower in the peach fruit treated with the sugars for $12 \mathrm{~h}$ and $24 \mathrm{~h}$ than they were in the control. All other metabolites had similar levels in both the sugar-treated and control peach fruit. These results indicated that glucose, sucrose, sorbitol, and fructose all had similar effects on metabolite accumulation in vitro. Moreover, after four sugars treatment for 12 and $24 \mathrm{~h}$, anthocyanins were not accumulated as cyanidin 3-O-glucoside, the main components in blood-flesh peach fruits, but as cyanidin-3-O-(6-O-p-coumaroyl) glucoside.

\subsection{Expression Levels of Genes Regulating the Anthocyanin Biosynthesis Pathway}

As anthocyanins accumulated in the flesh of the peach fruit treated with the sugars, we analyzed the expression of the genes involved in anthocyanin biosynthesis, based on the transcriptome data. Most of these genes were upregulated in the flesh of the peach fruit treated with sugars for $12 \mathrm{~h}$ and $24 \mathrm{~h}$ in vitro (Figure 2). The key structural genes PpDFR and P PUFGT were reported to be upregulated by the transcription factors PpMYB10.1 and PpBL, and their expression levels were also significantly higher in the flesh of the sugar-treated peach fruit compared with those of the control (Tables S5 and S6). By contrast, the key regulatory genes $P p M Y B 10.1$ and $P p B L$ were not upregulated in the flesh of the sugar-treated peach fruit compared with those of the control. In fact, $P p B L$ was downregulated in the flesh of the sugar-treated peach fruit (Tables S5 and S6). Further quantitative PCR verification was performed, and the results were similar as that of the transcriptome data. Hence, we speculated that other transcription factors might upregulate anthocyanin biosynthesis-related genes in response to sugar induction. 


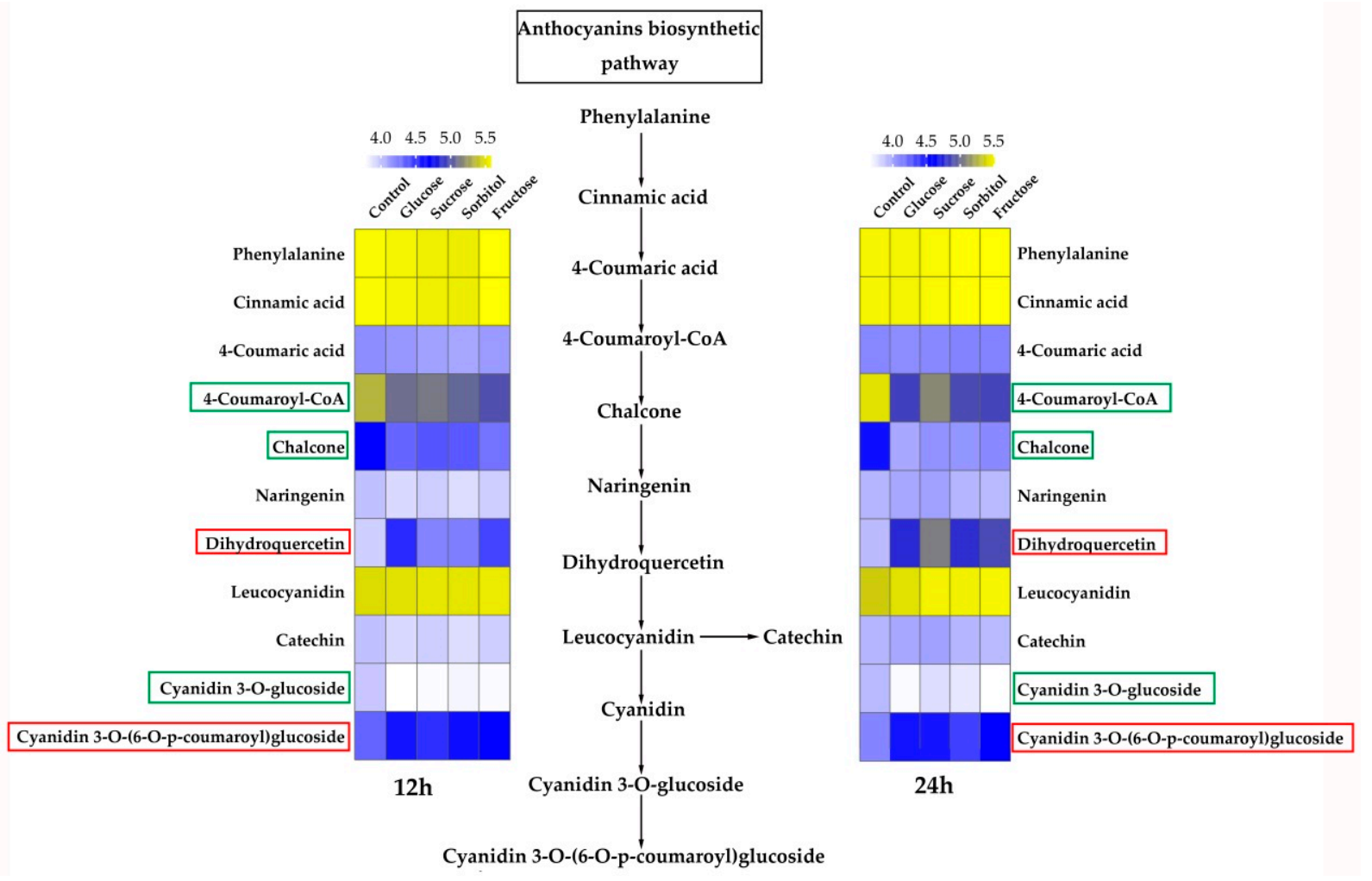

Figure 1. Metabolites involved in the anthocyanin biosynthesis pathway were determined in positive mode. Heat maps on the left and right indicate the metabolite content $(\mathrm{M} / \mathrm{Z})$ in the flesh of peach fruit treated with exogenous glucose, sucrose, sorbitol, and fructose for $12 \mathrm{~h}$ and $24 \mathrm{~h}$, respectively. The numbers at the top of the picture indicate $\log 10(\mathrm{M} / \mathrm{Z})$, which was listed in Tables S3 and S4. Metabolite names are shown on the side of the map. Metabolite content increases with blue and yellow color intensity in the square within the heat map. The red rectangle indicates that the metabolite content was higher in the flesh of the sugar-treated peach fruit than that of the untreated control. The green rectangle indicates that the metabolite content was lower in the flesh of the sugar-treated peach fruit than that of the untreated control.

2.3. Identification of Regulatory Genes Associated with High Expression Levels of Anthocyanin Biosynthesis-Related Genes in the Flesh of Sugar-Treated Peach Fruit

To identify the regulatory genes involved in anthocyanin biosynthesis in the flesh of sugar-treated peach fruit, we used the corresponding transcriptome data with fold change $>2.0$ in the analysis. The results indicated that 120,120,124, and 116 of the upregulated TFs were identified in the flesh of the peach fruit treated with glucose, sucrose, sorbitol, and fructose, respectively, for $12 \mathrm{~h}$. Then, 105 of these TFs were selected (Figure 3). Furthermore, $106,108,106$, and 114 of the upregulated TFs were identified in the flesh of the peach fruit treated with glucose, sucrose, sorbitol, and fructose, respectively, for $24 \mathrm{~h}$. Then, 98 of these TFs were selected, and 84 of the TFs common to both treatments had the potential in promoting anthocyanin biosynthesis, and these were thus selected for the subsequent analysis (Figure 4). Of these, the majority were WRKY- and EREBP-like gene family members and heat shock TFs ranked second. There were also eight MYB and bHLH gene family members. As MYB and bHLH family genes have been reported to regulate anthocyanin accumulation in the development of blood-flesh fruit [26], we selected two MYB genes designated PpMYB6 and PpMYB44-like and three bHLH genes designated PpbHLH35, PpbHLH51, and PpbHLH36-like according to their relative high expression to determine whether they regulate anthocyanin accumulation (Figure 5). 

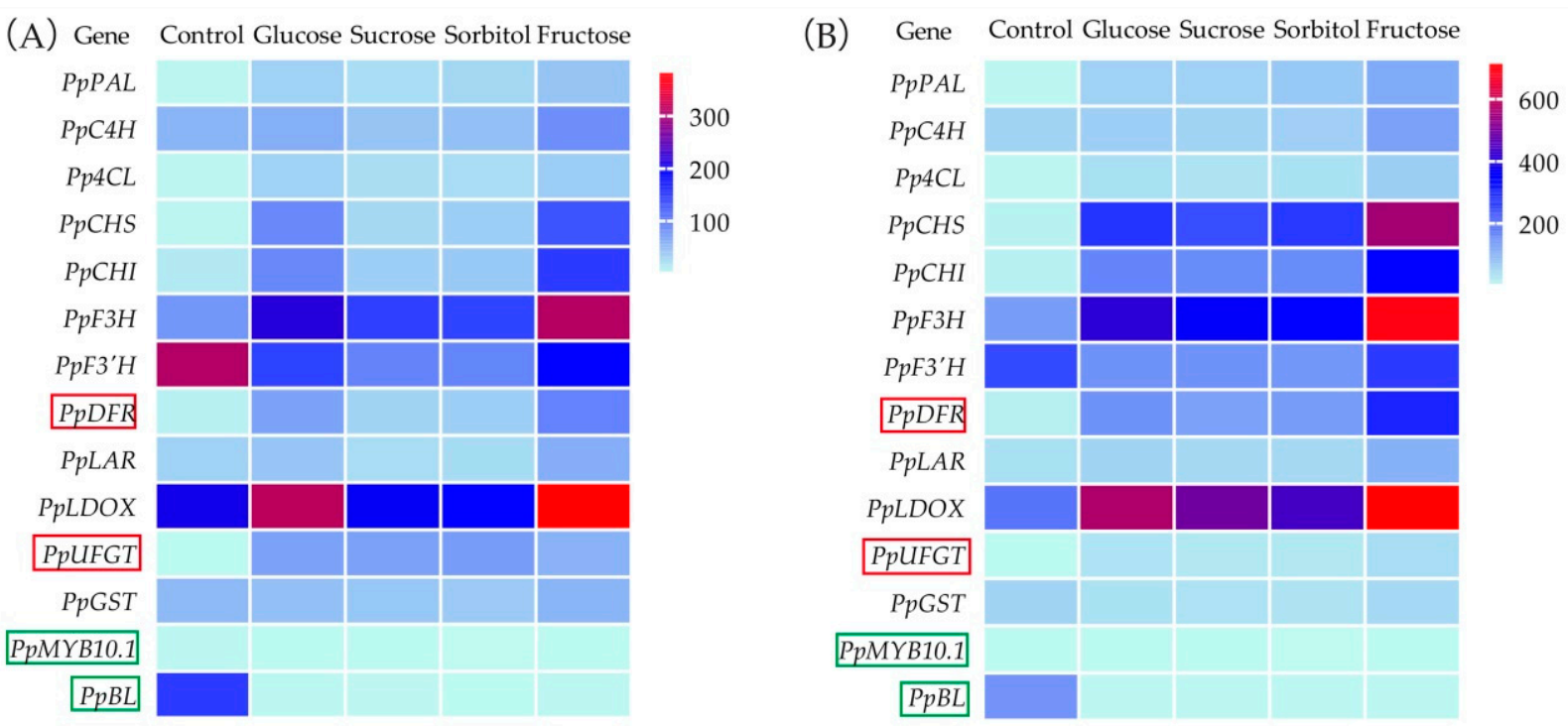

Figure 2. Heat map showing relative expression levels of anthocyanin biosynthesis and regulatory genes (FPKM) in flesh of sugar-treated and untreated peach fruit after $12 \mathrm{~h} \mathrm{(A)}$ and $24 \mathrm{~h}(\mathbf{B})$. Gene names are shown on left side of map. Gene expression level increases with blue and red color intensity in the square within the heat map. PpDFR and PpUFGT are in red in the box. PpMYB10.1 and PpBL are in green in the box.

(A)

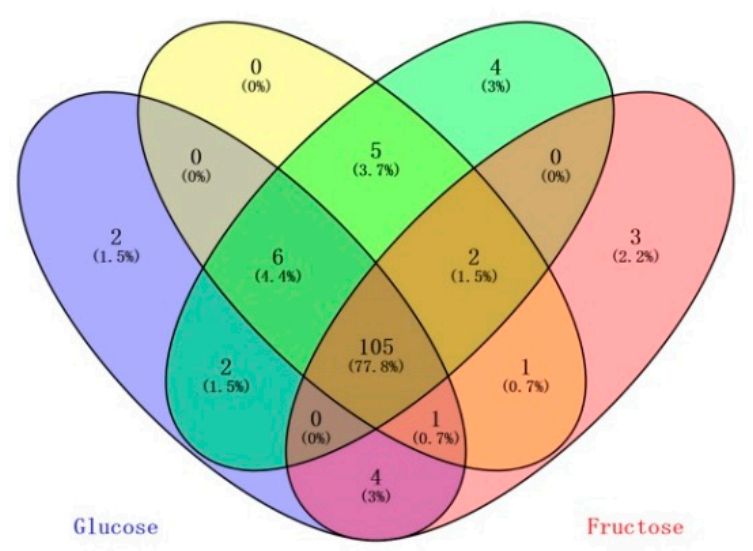

(B)

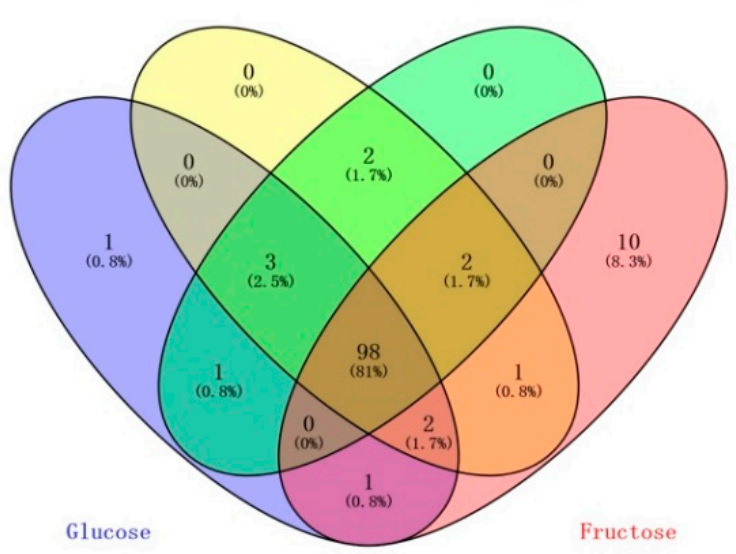

Figure 3. Venn diagrams showing numbers of upregulated TFs in flesh of glucose-, sucrose-, sorbitol-, and fructose-treated peach fruit after $12 \mathrm{~h} \mathrm{(A)}$ and $24 \mathrm{~h}(\mathbf{B})$.

\subsection{Prediction of the Selected MYB and bHLH TFs Using a Tobacco Leaf Dual Luciferase Assay}

To validate PpMYB6, PpMYB44-like, PpbHLH35, PpbHLH51, and PpbHLH36-like regulation in anthocyanin biosynthesis, we analyzed their phylogenetic relationships with other MYB and bHLH TFs that are known to upregulate this process (Figure 6A). PpMYB6 was phylogenetically related to OsMYB while Pp.8G134900 was more closely associated with PeMYB11. The bHLH TFs PpbHLH35, PpbHLH51, and PpbHLH36-like were clustered together and were near GhMYC1 (Figure 6B). Therefore, PpMYB6, Pp.8G134900, PpbHLH35, PpbHLH51, and PpbHLH36-like probably activated anthocyanin biosynthesis. 


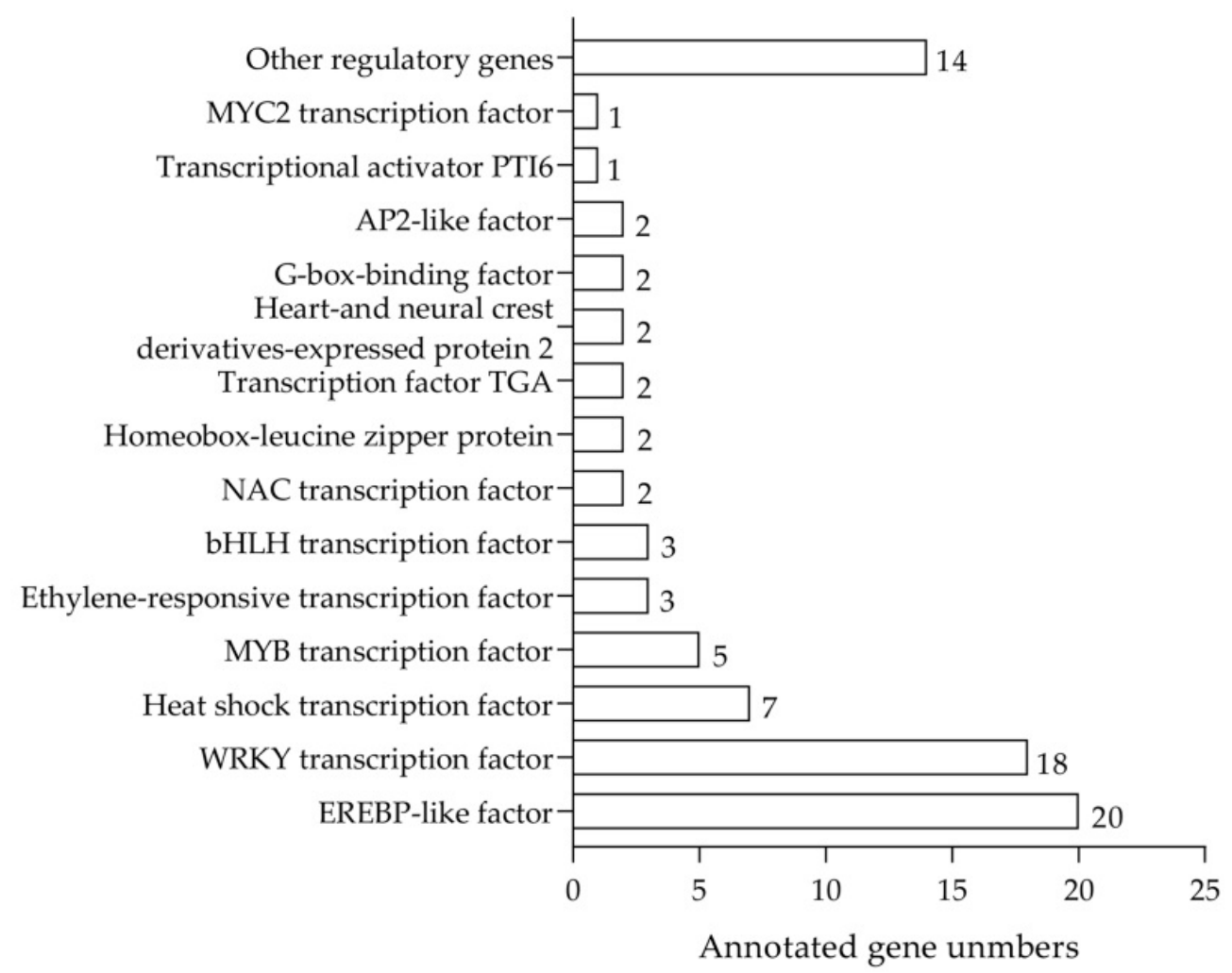

Figure 4. Eighty-four upregulated TFs common to flesh of peach fruit treated with glucose, sucrose, sorbitol, and fructose for $12 \mathrm{~h}$ and $24 \mathrm{~h}$.
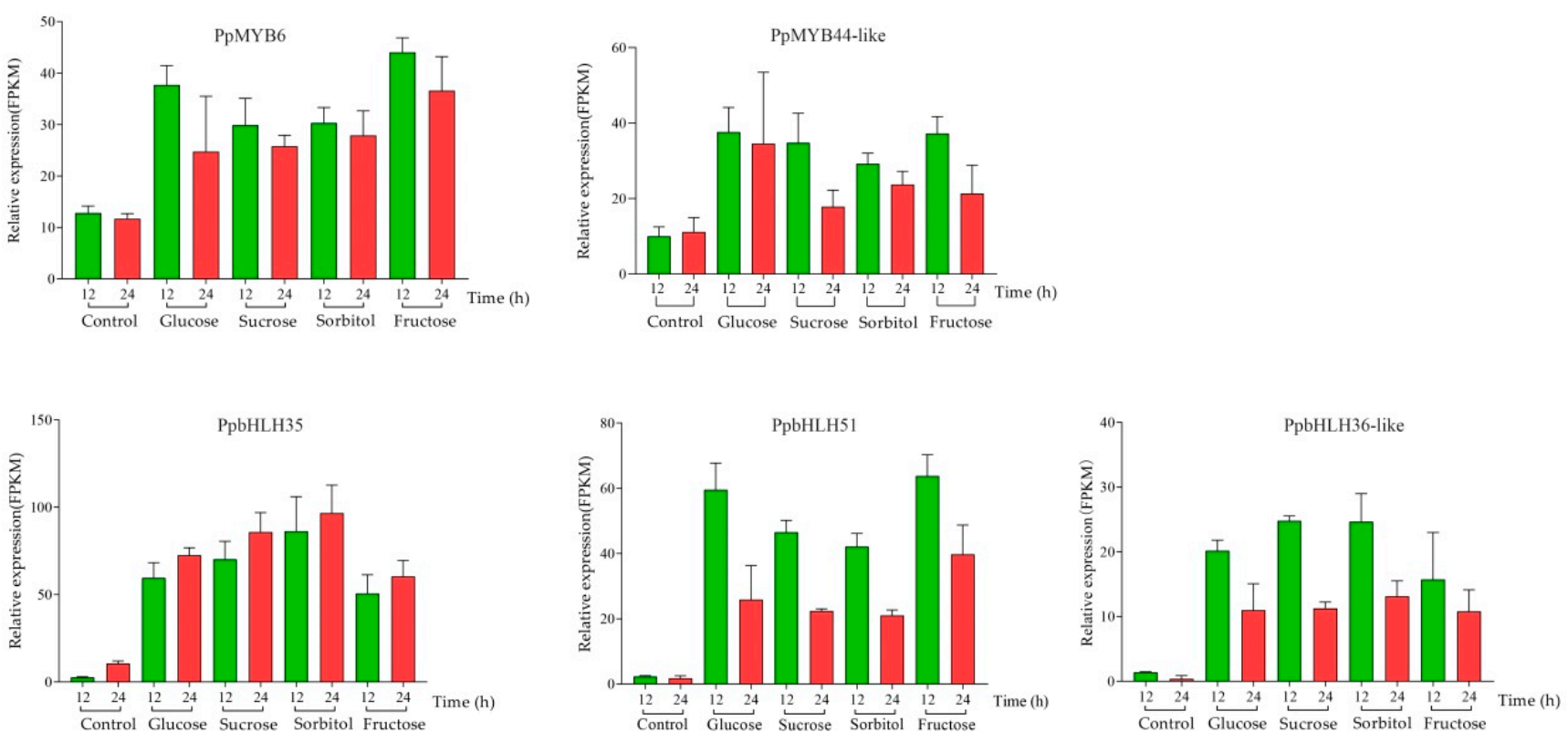

Figure 5. Relative expression levels of two MYB and three bHLH family genes selected among 84 upregulated TFs. 

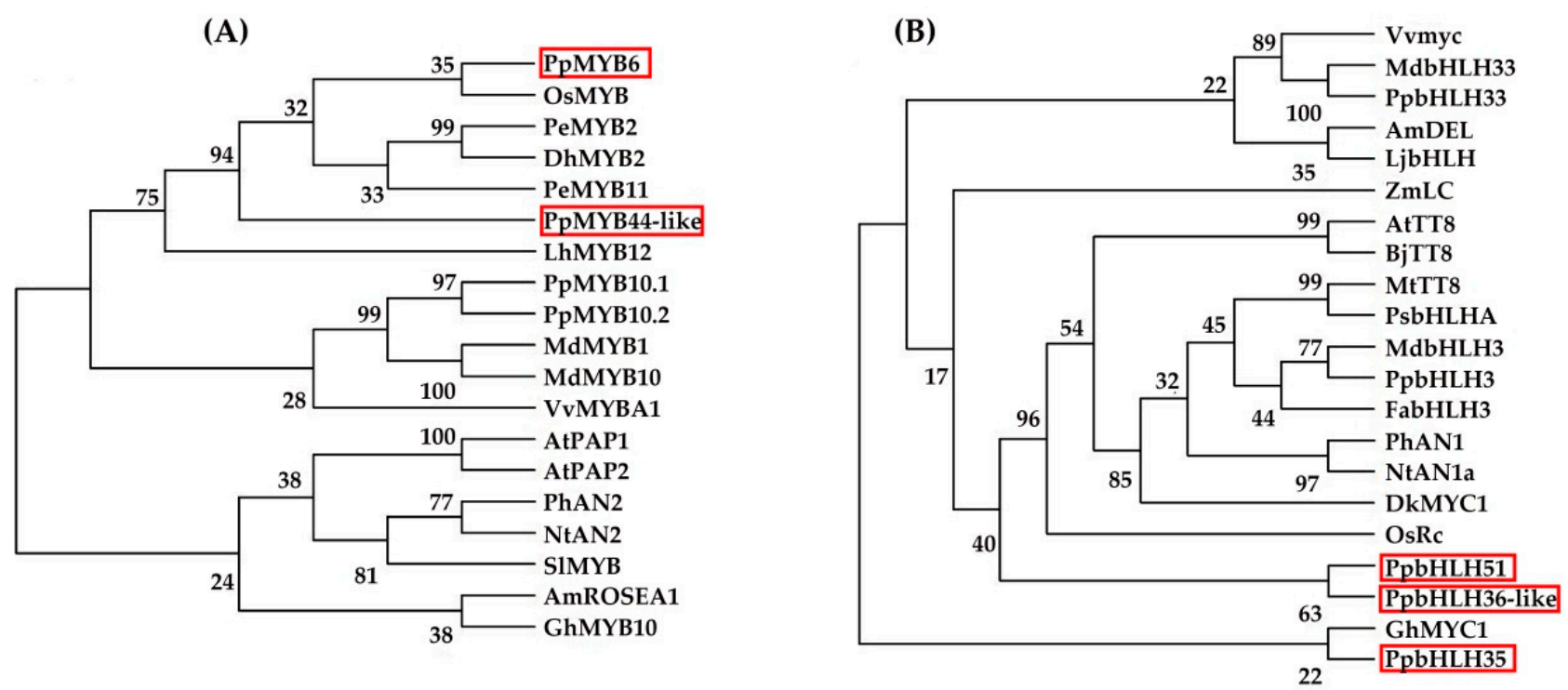

Figure 6. Phylogenetic analyses of amino acid sequences of MYB (A) and bHLH (B) TFs. Full MYB and bHLH TF amino acid sequence alignment and phylogenetic tree construction with MEGA-X software (Available online: https:/ / www.megasoftware.net/dload_win_gui (accessed on 21 June 2021)). Numbers before binary structures indicate bootstrap test results for 1000-replicate analysis. PpMYB6, PpMYB44-like, PpbHLH35, PpbHLH51, and PpbHLH36-like are highlighted by solid red rectangles. National Center for Biotechnology Information (NCBI) accession numbers are as follows: OsMYB (CAA45509); PeMYB2 (AIS35919); DhMYB2 (AQS79852); PeMYB11 (AIS35928); LhMYB12 (BAJ05398); MdMYB1 (ABK58138); MdMYB10 (ABB84753); VvMYBA1 (BAD18977); AtPAP1 (NP-176057); AtPAP2 (NP-176813); PhAN2 (AAF66727); NtAN2 (ACO52472); SIMYB (AAQ55181); AmROSEA1 (ABB83826); GhMYB10 (CAD87010); VvMYC (NP-001267954.1); MdbHLH33 (ABB84474.1); AmDEL (AAA32663.1); LjbHLH (BAJ10680.1); ZmLC (P13526.1); AtTT8 (OAO98324.1); BjTT8 (AIN41653.1); MtTT8 (AKN796061); PsbHLHA (E3SXU4.1); MdbHLH3 (ADL36597.1); FabHLH3 (AFL02463.1); PhAN1 (AAG259281); NtAN1a (AEE992571); DkMYC1 (AEC03343.1); OsRc (BAF42667); and GhMYC1 (CAA07615).

A dual luciferase assay was conducted on tobacco leaves that were transiently infiltrated with mixed GV3101 suspension. PpMYB6 activated the promoter PpUFGT when it was co-infiltrated with PpbHLH35, PpbHLH51, and PpbHLH36-like. When PpMYB44-like was co-infiltrated with PpbHLH35, it also activated PpUFGT. By contrast, PpMYB44-like did not activate PpUFGT even when it was co-infiltrated with PpbHLH51 and PpbHLH36-like (Figure 7). These results indicated that PpMYB6, PpMYB44-like, PpbHLH35, PpbHLH51, and PpbHLH36-like upregulation induced anthocyanin accumulation in the flesh of sugartreated peach fruit. 
(A)

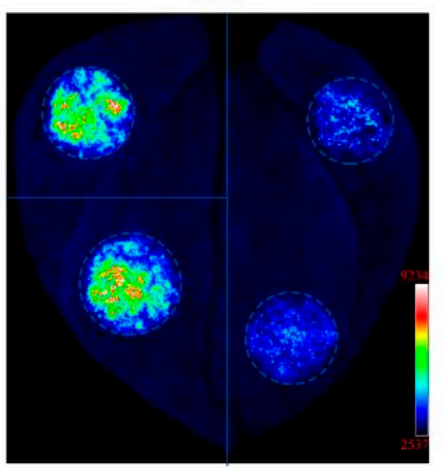

\begin{tabular}{|l|l|}
\hline $\begin{array}{l}\text { PpMYB6 } \\
\text { PpbHLH35 } \\
\text { proPpUFGT }\end{array}$ & GUS \\
\cline { 1 - 1 } PpMYB44-like & proUFGT \\
PpbHLH35 & \\
proUFGT & \\
\hline
\end{tabular}

(B)
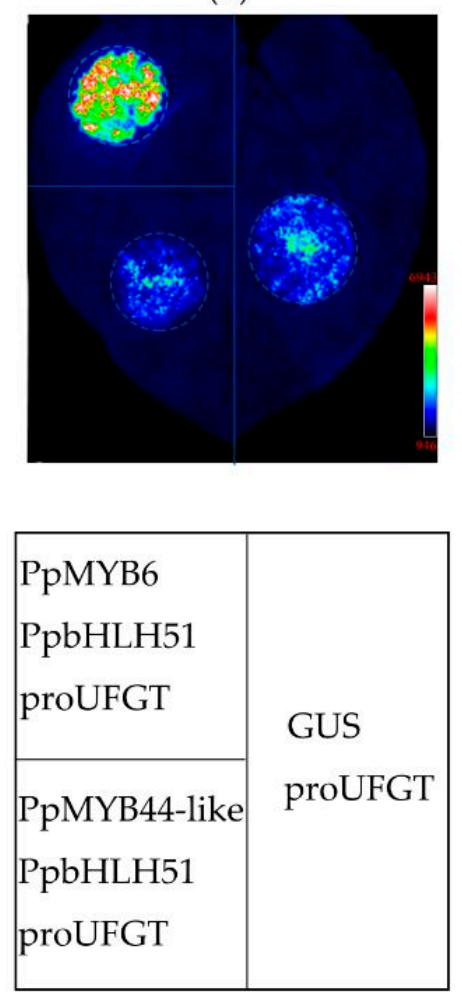

(C)
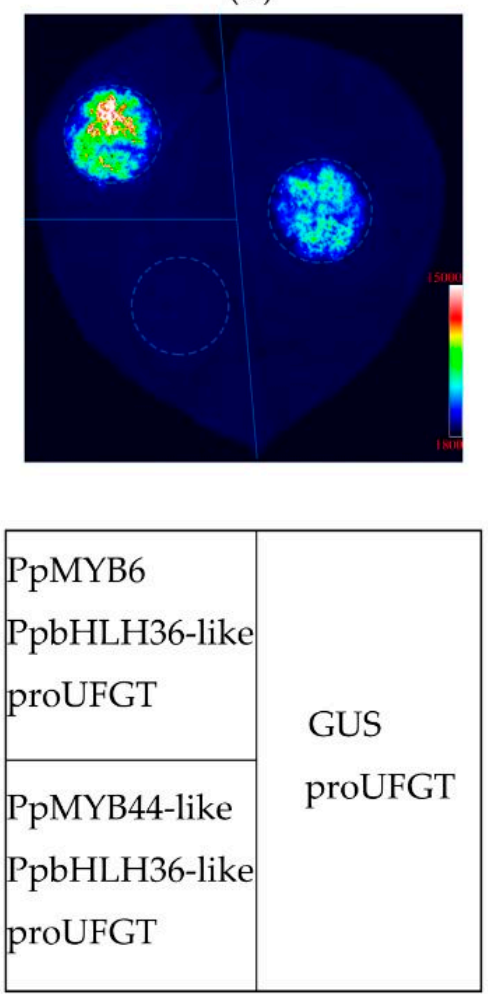

Figure 7. Regulatory relationships among selected MYB TFs and bHLH TFs and PpUFGT in tobacco leaf. Infiltration sites in upper left of leaf indicate combinations of 'PpMYB6, PpbHLH35, and proPpUFGT' (A), 'PpMYB6, PpbHLH51, and proPpUFGT' (B), and 'PpMYB6, PpbHLH36-like, and proPpUFGT' (C), respectively. Infiltration sites in lower left of leaf indicate combinations of 'PpMYB44-like, PpbHLH35, and proPpUFGT' (A), 'PpMYB44-like, PpbHLH51, and proPpUFGT' (B), and 'PpMYB44-like, PpbHLH36-like, and proPpUFGT' (C), respectively. A combination of 'GUS and proPpUFGT' was used as a negative control (right side of leaf). Color changed from blue to red indicates gradually increasing luciferase activity.

\section{Discussion}

Sugars promote anthocyanin accumulation in many plants. Cyanidin-3-glucoside was accumulated in Clematis pitcheri shoots in response to high sucrose concentrations [18]. Pelargonidin 3-glucoside, pelargonidin 3-rutinoside, pelargonidin 3-malonylglucoside, and pelargonidin 3-methylmalonyglucoside increased in postharvest strawberry fruit treated with $50 \mathrm{mM}$ sucrose [21]. Sucrose, glucose, fructose, and sorbitol induced similar degrees of cyanidin-3-O-rutinoside and cyanidin-3-O-glucoside accumulation in the fruit of the redblushed apricot cultivar [30]. Sucrose most strongly induced pelargonidin-3-O-glucoside accumulation in the hypocotyls and roots of red radish seedlings. By contrast, 1:1 fructose:glucose only weakly activated anthocyanin accumulation in the same crop [19]. The cyanidin-3-glucoside content increased in the mesocarp disks of blood-fleshed peach fruit treated with $100 \mathrm{mM}$ sucrose [29]. The foregoing reports demonstrated that anthocyanins accumulated as glycosides in fruit pulp. In this study, the glucose, sucrose, sorbitol, and fructose treatments all increased the relative anthocyanin content of blood-fleshed peach fruit. However, the anthocyanins accumulated in the form of cyanidin-3-O-(6-O-p-coumaroyl) glucoside rather than cyanidin 3-O-glucoside. After analyzing the transcriptome data, we found that there were three acyltransferase genes which might be associated with the acylation of ayanidin-3-O-glucoside (Tables S7 and S8). The peach cultivar, exogenous sugar concentrations, treatment times, and cultivation temperatures were similar between the present study and that of Rumainum et al. However, while we harvested our fruits before ripening, Rumainum et al. collected theirs at maturation. This sampling time discrepancy 
might account for the observed differences in anthocyanin accumulation between these studies.

Anthocyanin biosynthesis can be regulated by MYB and bHLH transcription factors (TFs). In Arabidopsis, AtPAP1, AtPAP2, AtMYB113, AtGL3, AtEGL3, and AtTT8 positively influenced anthocyanin biosynthesis $[9,17]$. In apple fruit, MdMYB10 promoted anthocyanin biosynthesis by interacting with both MdbHLH3 and MdbHLH33 [10]. In strawberry fruit, when FvMYB was co-infiltrated with FvbHLH33, it activated the FvDFR and FvUFGT promoters [16]. In peach fruit, PpMYB10.1, PpMYB10.2, PpMYB10.3, and Pp bHLH3 induced anthocyanin biosynthesis by upregulating the structural genes PpDFR and PpUFGT $[25,26]$. In the present study, two MYB TFs and three bHLH3 TFs were identified based on metabolome and transcriptome data. All of them were phylogenetically near OsMYB, PeMYB11, and GhMYC1 which upregulate anthocyanin biosynthesis [31-33]. Thus, they are probably anthocyanin biosynthesis activators in sugar-treated peach fruit flesh.

Arabidopsis uses different signal transduction pathways for sucrose and neutral sugars such as glucose and fructose. Glucose signaling molecules are sensed by the hexokinase HXK1 in Arabidopsis. HXK1 regulates the expression of sugar-related genes such as CAB1 (chlorophyll $a / b$-binding protein), $P C$ (plastocyanin), and $r b c S$ (ribulose-1,5-bisphosphate carboxylase small subunit) [34]. Sucrose represses ATB2/AtbZIP11 (leucine zipper (bZIP)type transcription factor) translation via an open reading frame (ORF) encoding 42 amino acids. By contrast, glucose and fructose are relatively less effective in this process [35]. Sucrose also upregulates certain genes controlling anthocyanin biosynthesis whereas glucose and fructose have weak or no impact on their expression levels [36]. However, we found that glucose, sucrose, fructose, and sorbitol all had similar effects on anthocyanin accumulation. Therefore, they might activate the anthocyanin biosynthesis-specific TFs, which further contribute to anthocyanin biosynthesis in peach fruit flesh.

As signaling molecules, sugars control regulatory genes associated with anthocyanin biosynthesis [37]. In Arabidopsis seedlings, the regulatory genes AtMYB75/AtPAP1 involved in anthocyanin biosynthesis are upregulated by sucrose induction [22]. This signaling system is induced independently of hexokinase (HXK1) systems. In apple fruit, the energy sensor MdSnRK1.1 interacts with MdJAZ18, which is a repressor in the jasmonate signaling pathway. MdJAZ18 is then phosphorylated and degraded, MdbHLH3 is released, and its sucrose-induced anthocyanin accumulation activity is recovered [38]. It has been reported that MdbHLH3 can interact with the MYB transcription factors MdMYB9 and MdMYB11 to facilitate anthocyanin biosynthesis in apple [39,40]. Hence, MdMYB9 and MdMYB11 might participate in sucrose-induced anthocyanin accumulation. In the present study, the MYB transcription factors PpMYB6 and PpMYB44-like and the bHLH transcription factors PpbHLH35, PpbHLH51, and PpbHLH36-like were identified and activated the expression of the key anthocyanin structural gene PpUFGT. Hence, the foregoing TFs have positive influences on anthocyanin accumulation. However, the key regulatory genes $P p M Y B 10.1$ and PpBL $[25,26]$ were not upregulated. By contrast, they were highly expressed at the late stage of blood-flesh peach fruit development. They participate in anthocyanin accumulation by upregulating the important structural genes PpDFR and PpUFGT. Thus, we propose that the regulatory mechanism of anthocyanin accumulation differs between fruits subjected to exogenous sugar treatment in vitro and those that naturally ripen on a tree.

Based on the foregoing findings, we propose a model for anthocyanin accumulation in the flesh of peach fruit treated with glucose, sucrose, sorbitol, and fructose in vitro (Figure 8). 


\section{Sugars signaling}

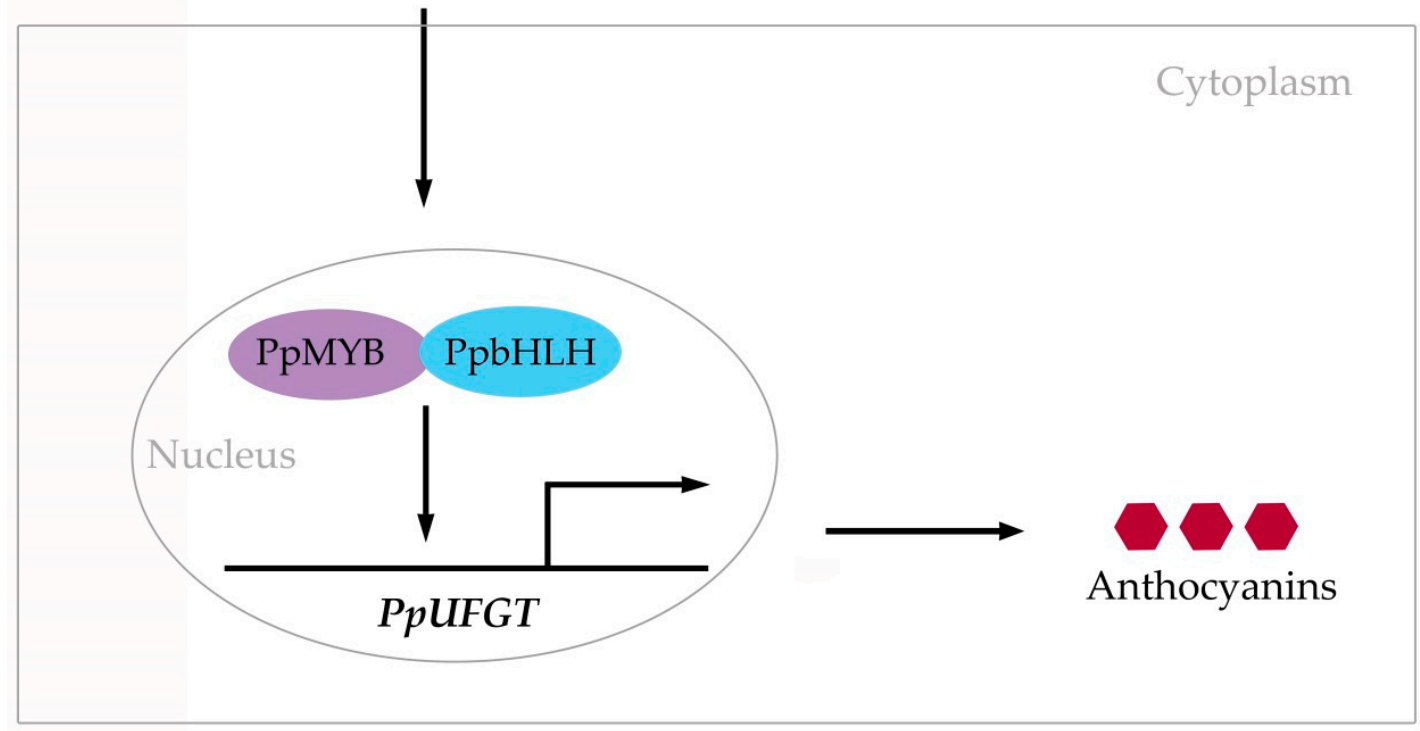

Figure 8. Model of two MYB and three bHLH3 TFs activating anthocyanin accumulation in flesh of peach fruit treated with four different sugars in vitro. Black arrow pointing to PpMYB and PpbHLH indicates that sugar signaling had a positive impact on their biosynthesis. Black arrow pointing to P PUFGT indicates that PpMYB and PpbHLH bind PpUFGT promoter. Folded line with arrow indicates that PpMYB and PpbHLH upregulate PpUFGT. Black arrow pointing to anthocyanins indicates that PpUFGT induces their biosynthesis. Sugar signaling was mediated by glucose, sucrose, sorbitol, and fructose. PpMYB includes PpMYB6 and PpMYB44-like. PpbHLH includes PpbHLH35, PpbHLH51, and PpbHLH36-like.

\section{Materials and Methods}

\subsection{Plant Materials}

The 14-year-old blood-fleshed peach cultivar 'Tianjin Shui Mi' was used in this study. It is an ancient Chinese landrace and contains abundant of anthocyanin compared with other cultivars. The anthocyanin content of this landrace is about 50 times that of other main cultivars. The trees were normally cultivated and managed. Ten fruits distributed along the outer crown of each tree were promptly picked at $80 \mathrm{~d}$ post-anthesis to measure their anthocyanin content in response to sugar treatment.

\subsection{Metabolite Production of Fruit Flesh Induced by Sugars In Vitro}

The fruit flesh was cut into small pieces $(2 \mathrm{~mm} \times 2 \mathrm{~mm} \times 2 \mathrm{~mm})$ and incubated at room temperature $\left(25^{\circ} \mathrm{C}\right)$ in 2-(4-morpholino)ethanesulfonic acid (MES) culture medium ( $\mathrm{pH}$ 6.5) containing various sugars (glucose, sucrose, fructose, or sorbitol) (Solarbio Beijing China). The control was sugar-free MES medium. The MES medium consisted of $100 \mathrm{mM}$ MES (pH 5.5), $5 \mathrm{mM} \mathrm{CaCl2,} 1 \mathrm{mM}$ EDTA, $10 \mathrm{mM}$ vitamin $\mathrm{C}$ (ascorbic acid), and $100 \mathrm{mM}$ glucose, sucrose, fructose, or sorbitol. After $12 \mathrm{~h}$ and $24 \mathrm{~h}$, peach fruit flesh was immersed in liquid nitrogen and stored at $-80^{\circ} \mathrm{C}$.

\subsection{Metabolomics Analysis}

Three replicates of the flesh from sugar-treated and the control peach fruit were used in the metabolomics analysis. Twenty-five milligrams of peach fruit flesh were placed in a $1.5 \mathrm{~mL}$ centrifuge tube (Eppendorf $\mathrm{GmbH}$, Hamburg, Germany) containing $800 \mu \mathrm{L}$ of an aqueous methanol solution and pulverized in TissueLyser (QIAGEN Shanghai China) with a steel ball at $55 \mathrm{~Hz}$ for $4 \mathrm{~min}$. The powder was then centrifuged at $30,000 \times g$ for $20 \mathrm{~min}$ and the supernatant was transferred to a new EP tube. The EP tube was placed in the LC-MS (liquid chromatograph-mass spectrometer) system (ACQUITY UPLC BEH 
C18; $100 \mathrm{~mm} \times 2.1 \mathrm{~mm} ; 1.7 \mathrm{~mm}$; Waters Corp., Milford, MA, USA) for reversed-phase separation. The column oven temperature was kept constant at $50{ }^{\circ} \mathrm{C}$, the flow rate was $0.4 \mathrm{~mL} / \mathrm{min}$, and the mobile phase consisted of solvent A (water $+0.1 \%(v / v)$ formic acid) and solvent $B$ (acetonitrile $+0.1 \%(v / v)$ formic acid). The gradient elution conditions were $100 \%$ phase A, $0-2 \mathrm{~min} ; 0-100 \%$ phase $\mathrm{B}, 2-11 \mathrm{~min} ; 100 \%$ phase $\mathrm{B}, 11-13 \mathrm{~min}$; and $0-100 \%$ phase A, 13-15 min. The sample injection volume was $10 \mu \mathrm{L}$ [41].

The metabolites eluted from the column were detected by mass spectrometry (Xevo G2 XS QTOF; Waters Corp.). The Q-TOF (quadrupole time-of-flight) was used in both positive and negative ion modes. The cone voltages were set to $3 \mathrm{kV}-40 \mathrm{~V}$ and $1 \mathrm{kV}-40 \mathrm{~V}$ for the positive and negative ion modes, respectively. The TOF mass arrangement was 50-1200 Da and the scan time was $0.2 \mathrm{~s}$. For MS/MS detection, all precursors were fragmented using 20-40 eV and the scan time was set to $0.2 \mathrm{~s}$. The MS data were acquired in centroid MSE mode and identified according to the Kyoto Encyclopedia of Genes and Genomes (KEGG) database. Metabolites differentially expressed between the sugar treatments and the control were selected based on the parameters fold-change (sugar treatment/control) $>1.2$ or $<0.8333$ [42].

\subsection{RNA Sequencing}

Total RNA was extracted with a kit (Waryong, Beijing, China), treated with RNasefree DNAase (Takara, Dalian, China), and reverse-transcribed with a Supremo III RT kit (BioTeKe, Beijing, China). Total RNA concentration and purity were assessed with an Agilent Bioanalyzer 2100 Bioanalyzer (Agilent Technologies, Santa Clara, CA, USA) and NanoDrop 2000 (Thermo, Waltham, MA, USA), respectively.

Magnetic beads with Oligo (dT)s were used to enrich mRNA from $5 \mathrm{mg}$ total RNA. The mRNA was randomly fragmented with fragmentation buffer and first-strand cDNA was synthesized with random hexamers. Double-stranded cDNA was then synthesized with dNTPs, RNase H, and DNA polymerase I. The double-stranded cDNA was enriched by adding poly-(A)s and PCR amplification. The enriched cDNA was linked to a vector, which was used to construct sequencing library, and analyzed in an Agilent Bioanalyzer 2100 system (Agilent Technologies). The cDNA library sequencing was performed in a HiSeq 2500 system (Illumina, San Diego, CA, USA). All peach fruit flesh samples were sequenced in three biological replicates. The low-quality reads were removed and the high-quality data were aligned to the peach reference genome (Lovell 2.0) with TopHat2 using its default parameters [43]. The gene expression levels were calculated as fragments per kilobase per million reads (FPKM). Gene ontology (GO) annotations were analyzed according to Blast2GO [44] and WEGO [45]. Differentially expressed upregulated genes were selected according to the criteria of sugar treatment FPKM $>1$ and fold change (sugar treatment/control) $>2.0$. The upregulated transcription factors (TFs) were selected for the samples of flesh of peach fruit exposed to the sugars for $12 \mathrm{~h}$. The TFs common to all four data types were screened. For the samples of peach fruit flesh exposed to the sugars for $24 \mathrm{~h}$, the upregulated TFs were selected in the same manner as those identified for the samples of peach fruit flesh exposed to the sugars for $12 \mathrm{~h}$. The upregulated TFs common to both foregoing treatments were selected for the subsequent analysis.

\subsection{Dual-Luciferase Tobacco Leaf Assay}

The promoter sequence of Pp.UFGT from 'Tianjin Shui Mi' was synthesized at Beijing Liuhe Bgi Co. Ltd. (Beijing, China) and infused into pGreenII 0800LUC vector [26,46]. The sequence of promoter of PpUFGT was taken from GDR database (Available online: https://www.rosaceae.org (accessed on 12 July 2021)). The coding sequences (CDS) of PpMYB6, PpMYB44-like, PpbHLH35, PpbHLH51, and PpbHLH36-like selected from the transcriptome analysis were also synthesized at Beijing Liuhe Bgi Co. Ltd. and inserted into the pBI121 vector under the control of the $35 \mathrm{~s}$ promoter. The GUS gene was infused into the pBI121 vector as a negative control [26]. All recombinant vectors were transformed into A. tumefaciens GV3101 and incubated at $28{ }^{\circ} \mathrm{C}$ for $2 \mathrm{~d}$. Individual transformants were resuspended in $1.0 \mathrm{~mL}$ Luria-Bertani (LB) medium (Solarbio Beijing China) containing 
$50 \mathrm{mg} / \mathrm{mL}$ kanamycin for $10 \mathrm{~h}$. Ten microliters were placed in $15 \mathrm{~mL}$ LB medium containing $50 \mathrm{mg} / \mathrm{mL}$ kanamycin and shaken at $28^{\circ} \mathrm{C}$ for $8-12 \mathrm{~h}$. After centrifuging and removing the medium, we adjusted the agrobacteria $O D$ to $0.4-0.6$ with infiltration buffer comprising 0.5 M MES, $1.0 \mathrm{mM} \mathrm{MgCl}_{2}$, and $1.0 \mu \mathrm{M}$ acetosyringone. The suspension was injected into three young leaves per tobacco (Nicotiana benthamiana) plant. The leaves injected with agrobacteria were excised after $2.5 \mathrm{~d}$, immersed in $D$-luciferin sodium salt solution for $10 \mathrm{~min}$, and placed in a multifunctional imaging analysis system (Tanon, Shanghai, China) to measure luminosity.

\section{Conclusions}

'PpMYB6 and PpbHLH35' ,PpMYB6 and PpbHLH51' , PpMYB6 and PpbHLH36-like', and 'PpMYB44-like and PpbHLH35' might participate in anthocyanin accumulation via upregulating key structural gene (PpUFGT) in the flesh of peach fruit treated with exogenous glucose, sucrose, sorbitol, and fructose in vitro.

Supplementary Materials: The following are available online at https://www.mdpi.com/article/10 $.3390 /$ plants11040507/s1. The metabolome and transcriptome analysis original data were deposited in the Figshare database (Available online: https:/ / doi.org/10.6084/m9.figshare.15071700.v1 (accessed on 15 September 2021)). Figure S1: Pathway enrichment analysis of top 100 upregulated metabolites in flesh of peach fruit treated with glucose, sucrose, sorbitol, and fructose for $12 \mathrm{~h}$, Figure S2: Pathway enrichment analysis of top 100 downregulated metabolites in flesh of peach fruit treated with glucose, sucrose, sorbitol, and fructose for 12 h, Figure S3: Pathway enrichment analysis of top 100 upregulated metabolites in flesh of peach fruit treated with glucose, sucrose, sorbitol, and fructose for $24 \mathrm{~h}$, Figure S4: Pathway enrichment analysis of top 100 downregulated metabolites in flesh of peach fruit treated with glucose, sucrose, sorbitol, and fructose for $24 \mathrm{~h}$, Table S1: Metabolites generated through LC-MC system in flesh of peach fruit treated with glucose, sucrose, sorbitol, and fructose for $12 \mathrm{~h}$, Table S2: Metabolites generated through LC-MC system in flesh of peach fruit treated with glucose, sucrose, sorbitol, and fructose for $24 \mathrm{~h}$, Table S3: Flavonoids in the anthocyanin pathway, which were identified in flesh of peach fruit treated with glucose, sucrose, sorbitol, and fructose for $12 \mathrm{~h}$, Table S4: Flavonoids in the anthocyanin pathway, which were identified in flesh of peach fruit treated with glucose, sucrose, sorbitol, and fructose for $24 \mathrm{~h}$. Table S5: Relative expression (FPKM) of major anthocyanin biosynthesis and regulatory genes in flesh of sugar-treated and untreated peach fruit after $12 \mathrm{~h}$, Table S6: Relative expression (FPKM) of major anthocyanin biosynthesis and regulatory genes in flesh of sugar-treated and untreated peach fruit after $24 \mathrm{~h}$, Table S7: Relative expression (FPKM) of acyltransferase genes which might be associated with the acylation of cyanidin 3-O-glucoside in sugar treated flesh for $12 \mathrm{~h}$, Table S8: Relative expression (FPKM) of acyltransferase genes which might be associated with the acylation of cyanidin 3-O-glucoside in sugar treated flesh for $24 \mathrm{~h}$, Tables S9-S24: Top 100 up or downregulated metabolites in flesh of peach fruit treated with glucose, sucrose, sorbitol, and fructose for 12 and $24 \mathrm{~h}$, respectively.

Author Contributions: Writing-original draft preparation, J.W.; methodology, K.C.; validation, L.W.; formal analysis, X.Z.; data curation, W.D.; writing-review and editing, W.L. All authors have read and agreed to the published version of the manuscript.

Funding: This study was financially supported by the National Key Research and Development Program (No. 2018YFD1000200) and the Agricultural Science and Technology Innovation Program (No. CAAS-ASTIP-2016-ZFRI-01.

Institutional Review Board Statement: Not applicable.

Informed Consent Statement: Not applicable.

Data Availability Statement: Data will be available on reasonable request.

Conflicts of Interest: The authors declare no conflict of interest. 


\section{References}

1. Jaakola, L. New insights into the regulation of anthocyanin biosynthesis in fruits. Trends Plant Sci. 2013, 18, 477-483. [CrossRef] [PubMed]

2. Ogata, J.; Kanno, Y.; Itoh, Y.; Tsugawa, H.; Suzuki, M. Plant biochemistry: Anthocyanin biosynthesis in roses. Nature 2005, 435, 757-758. [CrossRef] [PubMed]

3. Cooper-Driver, G.A. Contributions of Jeffrey Harborne and co-workers to the study of anthocyanins. Phytochemistry 2001, 56, 229-236. [CrossRef]

4. Kong, J.M.; Chia, L.S.; Goh, N.K.; Chia, T.F.; Brouillard, R. Analysis and biological activities of anthocyanins. Phytochemistry 2003, 64, 923-933. [CrossRef]

5. Pojer, E.; Mattivi, F.; Johnson, D.; Stockley, C.S. The Case for Anthocyanin Consumption to Promote Human Health: A Review. Compr. Rev. Food Sci. Food Saf. 2013, 12, 483-508. [CrossRef]

6. Winkel-Shirley, B. Flavonoid biosynthesis. A colorful model for genetics, biochemistry, cell biology, and biotechnology. Plant Physiol. 2001, 126, 485-493. [CrossRef]

7. Tohge, T.; Fernie, A.R. Leveraging Natural Variance towards Enhanced Understanding of Phytochemical Sunscreens. Trends Plant Sci. 2017, 22, 308-315. [CrossRef]

8. Grotewold, E. The genetics and biochemistry of floral pigments. Annu. Rev. Plant Biol. 2006, 57, 761-780. [CrossRef]

9. Gonzalez, A.; Zhao, M.; Leavitt, J.M.; Lloyd, A.M. Regulation of the anthocyanin biosynthetic pathway by the TTG1/bHLH/Myb transcriptional complex in Arabidopsis seedlings. Plant J. Cell Mol. Biol. 2008, 53, 814-827. [CrossRef]

10. Espley, R.V.; Brendolise, C.; Chagné, D.; Kutty-Amma, S.; Green, S.; Volz, R.; Putterill, J.; Schouten, H.J.; Gardiner, S.E.; Hellens, R.P.; et al. Multiple repeats of a promoter segment causes transcription factor autoregulation in red apples. Plant Cell 2009, 21, 168-183. [CrossRef]

11. Kobayashi, S.; Goto-Yamamoto, N.; Hirochika, H. Retrotransposon-induced mutations in grape skin color. Science 2004, 304,982 [CrossRef] [PubMed]

12. Walker, A.R.; Lee, E.; Bogs, J.; McDavid, D.A.; Thomas, M.R.; Robinson, S.P. White grapes arose through the mutation of two similar and adjacent regulatory genes. Plant J. Cell Mol. Biol. 2007, 49, 772-785. [CrossRef] [PubMed]

13. Butelli, E.; Licciardello, C.; Zhang, Y.; Liu, J.; Mackay, S.; Bailey, P.; Reforgiato-Recupero, G.; Martin, C. Retrotransposons control fruit-specific, cold-dependent accumulation of anthocyanins in blood oranges. Plant Cell 2012, 24, 1242-1255. [CrossRef] [PubMed]

14. Feng, S.Q.; Wang, Y.L.; Yang, S.; Xu, Y.T.; Chen, X.S. Anthocyanin biosynthesis in pears is regulated by a R2R3-MYB transcription factor PyMYB10. Planta 2010, 232, 245-255. [CrossRef] [PubMed]

15. Yao, G.F.; Ming, M.L.; Allan, A.C.; Gu, C.; Li, L.T.; Wu, X.; Wang, R.Z.; Chang, Y.J.; Qi, K.J.; Zhang, S.L.; et al. Map-based cloning of the pear gene MYB114 identifies an interaction with other transcription factors to coordinately regulate fruit anthocyanin biosynthesis. Plant J. Cell Mol. Biol. 2017, 92, 437-451. [CrossRef] [PubMed]

16. Lin-Wang, K.; McGhie, T.K.; Wang, M.; Liu, Y.; Warren, B.; Storey, R.; Espley, R.V.; Allan, A.C. Engineering the anthocyanin regulatory complex of strawberry (Fragaria vesca). Front. Plant Sci. 2014, 5, 651. [CrossRef] [PubMed]

17. Borevitz, J.O.; Xia, Y.; Blount, J.; Dixon, R.A.; Lamb, C. Activation tagging identifies a conserved MYB regulator of phenylpropanoid biosynthesis. Plant Cell 2000, 12, 2383-2394. [CrossRef]

18. Kawa-Miszczak, L.; Wegrzynowicz-Lesiak, E.; Gabryszewska, E.; Saniewski, M. Effect of different sucrose and nitrogen levels in the medium on chlorophyll and anthocyanin content in Clematis pitcheri shoots cultured in vitro at different temperatures. $J$. Fruit Ornam. Plant Res. 2009, 17, 113-121.

19. Wang, L.; Jing, X.X.; Zhao, P.P.; Wang, L.F.; Yin, Y.; Li, Y.F. Simultaneous induction of anthocyanin and peroxidase by sucrose in hypocotyls and roots of Chinese red radish seedlings. Biol. Plant. 2020, 64, 828-837. [CrossRef]

20. Kopjar, M.; Tiban, N.N.; Pilizota, V.; Babic, J. Stability of anthocyanins, phenols and free radical scavenging activity through sugar addition during frozen storage of blackberries. J. Food Process. Preserv. 2010, 33, 1-11. [CrossRef]

21. Li, Z.B.; Wei, Y.Y.; Xu, Y.Y.; Han, P.P.; Jiang, S.; Xu, F.; Wang, H.F.; Tao, N.G.; Shao, X.F. Terpinen-4-ol treatment maintains quality of strawberry fruit during storage by regulating sucrose-induced anthocyanin accumulation. Postharvest Biol. Technol. 2021, 174, 111461. [CrossRef]

22. Teng, S.; Keurentjes, J.; Bentsink, L.; Koornneef, M.; Smeekens, S. Sucrose-specific induction of anthocyanin biosynthesis in Arabidopsis requires the MYB75/PAP1 gene. Plant Physiol. 2005, 139, 1840-1852. [CrossRef] [PubMed]

23. Shen, Z.J.; Confolent, C.; Lambert, P.; Poessel, J.-L.; Quilot-Turion, B.; Yu, M.; Ma, R.J.; Pascal, T. Characterization and genetic mapping of a new blood-flesh trait controlled by the single dominant locus DBF in peach. Tree Genet. Genom. 2013, 9, 1435-1446. [CrossRef]

24. Jiao, Y.; Ma, R.J.; Shen, Z.J.; Yan, J.; Yu, M.L. Gene regulation of anthocyanin biosynthesis in two blood-flesh peach (Prunus persica (L.) Batsch) cultivars during fruit development. J. Zhejiang Univ. Sci. B 2014, 15, 809-819. [CrossRef] [PubMed]

25. Rahim, M.A.; Busatto, N.; Trainotti, L. Regulation of anthocyanin biosynthesis in peach fruits. Planta 2014, 240, 913-929. [CrossRef] [PubMed]

26. Zhou, H.; Lin-Wang, K.; Wang, H.L.; Gu, C.; Dare, A.P.; Espley, R.V.; He, H.P.; Allan, A.C.; Han, Y.P. Molecular genetics of blood-fleshed peach reveals activation of anthocyanin biosynthesis by NAC transcription factors. Plant J. Cell Mol. Biol. 2015, 82, 105-121. [CrossRef] 
27. Font, I.; Gogorcena, M.A. Fruit sugar profile and antioxidants of peach and nectarine cultivars on almond $\times$ peach hybrid rootstocks. Sci. Hortic. 2013, 164, 563-572. [CrossRef]

28. Cordts, J.M.; Scorza, R.; Bell, R.L. Effects of carbohydrates and nitrogen on the development of anthocyanins of a red leaf peach (Prunus persica (L.) Batsch) in vitro. Plant Cell Tissue Organ Cult. 1987, 9, 103-110. [CrossRef]

29. Rumainum, I.M.; Worarad, K.; Yamaki, Y.; Yamane, K. Effects of Sucrose and Plant Hormone on the Pigmentation of Mesocarp of White- and Red-Fleshed Peach Fruits. Agrivita 2018, 40, 202-211. [CrossRef]

30. Huang, Z.Y.; Wang, Q.H.; Xia, L.H.; Hui, J.T.; Li, J.X.; Feng, Y.B.; Chen, Y.L. Preliminarily exploring of the association between sugars and anthocyanin accumulation in apricot fruit during ripening. Sci. Hortic. 2019, 248, 112-117. [CrossRef]

31. Elomaa, P.; Mehto, M.; Kotilainen, M.; Helariutta, Y.; Nevalainen, L.; Teeri, T.H. A bHLH transcription factor mediates organ, region and flower type specific signals on dihydroflavonol-4-reductase (dfr) gene expression in the inflorescence of Gerbera hybrida (Asteraceae). Plant J. Cell Mol. Biol. 1998, 16, 93-99. [CrossRef] [PubMed]

32. Hsu, C.C.; Chen, Y.Y.; Tsai, W.C.; Chen, W.H.; Chen, H.H. Three R2R3-MYB transcription factors regulate distinct floral pigmentation patterning in Phalaenopsis spp. Plant Physiol. 2015, 168, 175-191. [CrossRef] [PubMed]

33. Zhao, S.S.; Wang, C.H.; Ma, J.; Wang, S.; Tian, P.; Wang, J.L.; Cheng, Z.J.; Zhang, X.; Guo, X.P.; Li, C.L. Map-based cloning and functional analysis of the chromogen gene $C$ in rice (Oryza sativa L.). J. Plant Biol. 2016, 59, 496-505. [CrossRef]

34. Moore, B.; Zhou, L.; Rolland, F.; Hall, Q.; Cheng, W.H.; Liu, Y.X.; Hwang, I.; Jones, T.; Sheen, J. Role of the Arabidopsis glucose sensor HXK1 in nutrient, light, and hormonal signaling. Science 2003, 300, 332-336. [CrossRef] [PubMed]

35. Wiese, A.; Elzinga, N.; Wobbes, B.; Smeekens, S. A conserved upstream open reading frame mediates sucrose-induced repression of translation. Plant Cell 2004, 16, 1717-1729. [CrossRef] [PubMed]

36. Solfanelli, C.; Poggi, A.; Loreti, E.; Alpi, A.; Perata, P. Sucrose-specific induction of the anthocyanin biosynthetic pathway in Arabidopsis. Plant Physiol. 2006, 140, 637-646. [CrossRef] [PubMed]

37. Koch, K.E. Carbohydrate-modulated gene expression in plants. Annu. Rev. Plant Physiol. Plant Mol. Biol. 1996, 47, 509-540. [CrossRef]

38. Liu, X.J.; An, X.H.; Liu, X.; Hu, D.G.; Wang, X.F.; You, C.X.; Hao, Y.J. MdSnRK1.1 interacts with MdJAZ18 to regulate sucroseinduced anthocyanin and proanthocyanidin accumulation in apple. J. Exp. Bot. 2017, 68, 2977-2990. [CrossRef]

39. An, X.H.; Tian, Y.; Chen, K.Q.; Liu, X.J.; Liu, D.D.; Xie, X.B.; Cheng, C.G.; Cong, P.H.; Hao, Y.J. MdMYB9 and MdMYB11 are involved in the regulation of the JA-induced biosynthesis of anthocyanin and proanthocyanidin in apples. Plant Cell Physiol. 2015, 56, 650-662. [CrossRef]

40. Xie, X.B.; Li, S.; Zhang, R.F.; Zhao, J.; Chen, Y.C.; Zhao, Q.; Yao, Y.X.; You, C.X.; Zhang, X.S.; Hao, Y.J. The bHLH transcription factor MdbHLH3 promotes anthocyanin accumulation and fruit colouration in response to low temperature in apples. Plant Cell Environ. 2012, 35, 1884-1897. [CrossRef]

41. Peng, Z.; Gao, Q.; Luo, C.; Gong, W.F.; Tang, S.W.; Zhang, X.M.; Song, W.; Wang, Z.Z.; Liu, H.L.; Du, X.M.; et al. Flavonoid biosynthetic and starch and sucrose metabolic pathways are involved in the pigmentation of naturally brown-colored cotton fibers-ScienceDirect. Ind. Crops Prod. 2020, 158, 113045. [CrossRef]

42. Gao, H.Y.; Xu, D.Y.; Zhang, H.J.; Qian, J.P.; Yang, Q. Transcriptomics and metabolomics analyses reveal the differential accumulation of phenylpropanoids between Cinnamomum cassia Presl and Cinnamomum cassia Presl var. macrophyllum Chu. Ind. Crops Prod. 2020, 148, 112282. [CrossRef]

43. Kim, D.; Pertea, G.; Trapnell, C.; Pimentel, H.; Kelley, R.; Salzberg, S.L. TopHat2: Accurate alignment of transcriptomes in the presence of insertions, deletions and gene fusions. Genome Biol. 2013, 14, R36. [CrossRef] [PubMed]

44. Conesa, A.; Götz, S. Blast2GO: A comprehensive suite for functional analysis in plant genomics. Int. J. Plant Genom. 2008, 2008, 619832. [CrossRef]

45. Ye, J.; Fang, L.; Zheng, H.J.; Zhang, Y.; Chen, J.; Zhang, Z.J.; Wang, J.; Li, S.T.; Li, R.Q.; Bolund, L.; et al. WEGO: A web tool for plotting GO annotations. Nucleic Acids Res. 2006, 34, W293-W297. [CrossRef]

46. Hellens, R.P.; Allan, A.C.; Friel, E.N.; Bolitho, K.; Grafton, K.; Templeton, M.D.; Karunairetnam, S.; Gleave, A.P.; Laing, W.A Transient expression vectors for functional genomics, quantification of promoter activity and RNA silencing in plants. Plant Methods 2005, 1, 13. [CrossRef] 